\title{
Decreased renal function in overweight and obese prepubertal children
}

\author{
Liane Correia-Costa 1,2, Alberto Caldas Afonso ${ }^{1,2}$, Franz Schaefer ${ }^{3}$, João Tiago Guimarães ${ }^{1,4}$, Manuela Bustorff ${ }^{5}$, António Guerra ${ }^{6}$, \\ Henrique Barros ${ }^{1,7}$ and Ana Azevedo 1,7
}

BACKGROUND: Obesity is a potentially modifiable risk factor for the development and progression of kidney disease, both in adults and children. We aim to study the association of obesity and renal function in children, by comparing estimated glomerular filtration rate (eGFR) in nonoverweight and overweight/obese children. Secondarily, we aim to evaluate the accuracy of equations on eGFR estimation when compared to 24-h urinary creatinine clearance $(\mathrm{CrCl})$.

METHODS: Cross-sectional study of 313 children aged 8-9 y, followed in the birth cohort Generation XXI (Portugal). Creatinine and cystatin C, GFR estimated by several formulas and $\mathrm{CrCl}$ were compared in 163 nonoverweight and 150 overweight/obese, according to World Health Organization growth reference.

RESULTS: Overweight/obese children had significantly lower eGFR, estimated by all methods, except for $\mathrm{CrCl}$ and revised Schwartz formula. Despite all children having renal function in the normal range, eGFR decreased significantly with BMI $z$-score (differences ranging from -4.3 to $-1.1 \mathrm{ml} / \mathrm{min} / 1.73 \mathrm{~m}^{2}$ per standard deviation of BMI). The Zappitelli combined formula presented the closest performance to $\mathrm{CrCl}$, with higher correlation coefficients and higher accuracy values.

CONCLUSION: Young prepubertal children with overweight/ obesity already present significantly lower GFR estimations that likely represent some degree of renal impairment associated with the complex deleterious effects of adiposity.

$\mathbf{T}$ he role of the obesity epidemic in the risk of kidney disease has been recognized in adults, independently of diabetes, with the prevalence of end-stage renal disease raising tremendously in the past 3 decades in parallel with the increased prevalence of obesity $(1,2)$. Obesity was also identified as a strong and potentially modifiable risk factor for the development and progression of kidney disease $(3,4)$ and, in children, recent data confirmed a similar trend, with obesity contributing to renal injury and to an important increase of chronic kidney disease (5).
Studies in the general pediatric population only recently started to arise and the results concerning glomerular filtration rate (eGFR) differences in obese and normal weight children are somehow contradictory; some studies reported higher eGFR in obese children reflecting a state of hyperfiltration (6), while others found either the opposite $(7,8)$ or no differences $(9,10)$.

It is difficult to evaluate renal function in the general pediatric population and to which extent the kidney is involved in the metabolic cluster of conditions that emerge with the onset of obesity in early ages. On the one hand, since exogenous and more accurate, but invasive, methods are not justifiable to apply in healthy children, one has to rely on eGFR formulas or in the 24-h urine creatinine clearance $(\mathrm{CrCl})$. However, formulas to estimate eGFR are known to have some limitations and 24-h urine samples might be difficult to reliably obtain in children (11). On the other hand, the degree of kidney involvement related to obesity might be subtle, below the threshold to translate into changes in classic markers. The emergence of cystatin C (CysC) was expected to overcome some of the limitations of creatinine-based estimations, adding an improved clinical sensitivity in early renal damage (12). Though, studies in adults showed that $\mathrm{CysC}$ levels are increased in obese subjects $(13,14)$ and that CysC-based formulas may result in an underestimation of GFR in patients with higher BMI (15). Few studies exist on CysC levels in children, leaving a large uncertainty regarding the influence of body composition in the interpretation of CysC levels (16).

Our main objective was to compare renal function between nonoverweight and overweight/obese 8-9-y-old children, assessed by serum creatinine and $\mathrm{CysC}$, and eGFR, estimated by creatinine-based, CysC-based and combined formulas (including both creatinine and $\mathrm{CysC}$ ) and by $\mathrm{CrCl}$. Secondarily, we aimed to clarify if putative differences in renal function between groups reflect alterations in the kidney or are solely determined by nonrenal differences in direct association with body composition. We also aimed to evaluate the accuracy of selected equations of eGFR estimation when compared to $\mathrm{CrCl}$, in nonoverweight and overweight/obese children. 


\section{RESULTS}

A total of 313 children (53\% male) with a mean (SD) age of $8.8(0.2)$ y were included in the present analysis. Clinical characteristics and levels of biochemical parameters of all study subjects and separately for nonoverweight $(n=163)$ and overweight/obese children ( $n=150$, of whom 89 overweight and 61 obese) are shown in Table 1. The overweight/obese group presented higher blood pressure values and both systolic and diastolic blood pressure were significantly correlated with BMI z-score $(r=0.375, P<0.001$ and $r=0.162, P<0.001$, respectively) and with waist-to-height ratio (WHtR) $(r=0.315, P<$ 0.001 and $r=0.139, P=0.014$, respectively). Comparing renal function between the groups, both creatinine and CysC were significantly higher in the overweight/obese group. Depending on the method of estimation, the mean eGFR ranged from 113 to 162 in nonoverweight children and from 110 to $156 \mathrm{ml} /$ $\mathrm{min} / 1.73 \mathrm{~m}^{2}$ in overweight/obese children, with overweight/ obese children presenting significantly lower eGFR when estimated by all methods, except for $\mathrm{CrCl}$ and Schwartz-R formula (Table 1). No significant differences were found in the $24-\mathrm{h}$ urine albumin or protein excretion.
All children had renal function markers and estimated eGFR in the normal range (at or above $90 \mathrm{ml} / \mathrm{min} / 1.73 \mathrm{~m}^{2}$ ). Significant positive correlations were found between BMI $\mathrm{z}$-score and both creatinine $(r=0.18, P=0.002)$ and CysC $(r=0.29, P<0.001)$. Significant negative correlations were found between BMI z-score and estimations of eGFR, except for Schwartz-R. The strongest correlations were observed with the CysC-based equation $(r=-0.30, P<0.001)$, followed by Zappitelli-Comb formula $(r=-0.22, P<0.001$; Figure 1). In the age- and sex-adjusted linear regression analysis of markers of renal function on BMI z-score (Table 2), eGFR decreased significantly with BMI (except for Schwartz-R), with differences for the several methods ranging from -4.26 to $-1.10 \mathrm{ml} /$ $\mathrm{min} / 1.73 \mathrm{~m}^{2}$ per SD of BMI (1 SD BMI z-score is $\sim 1.2 \mathrm{~kg} / \mathrm{m}^{2}$ for girls and boys at this age).

Since significant differences in eGFR between nonoverweight and overweight/obese children were observed for all estimations including $\mathrm{Cys} \mathrm{C}$, and considering that obesity might be a nonrenal determinant of $\mathrm{Cys} C$, we quantified the independent association of $\mathrm{CysC}$ with $\mathrm{BMI} \mathrm{z}$-score and the creatinine-based methods to assess renal function. Both BMI

Table 1. General characteristics, renal function markers, and estimated glomerular filtration rates of the nonoverweight and overweight/obesity groups

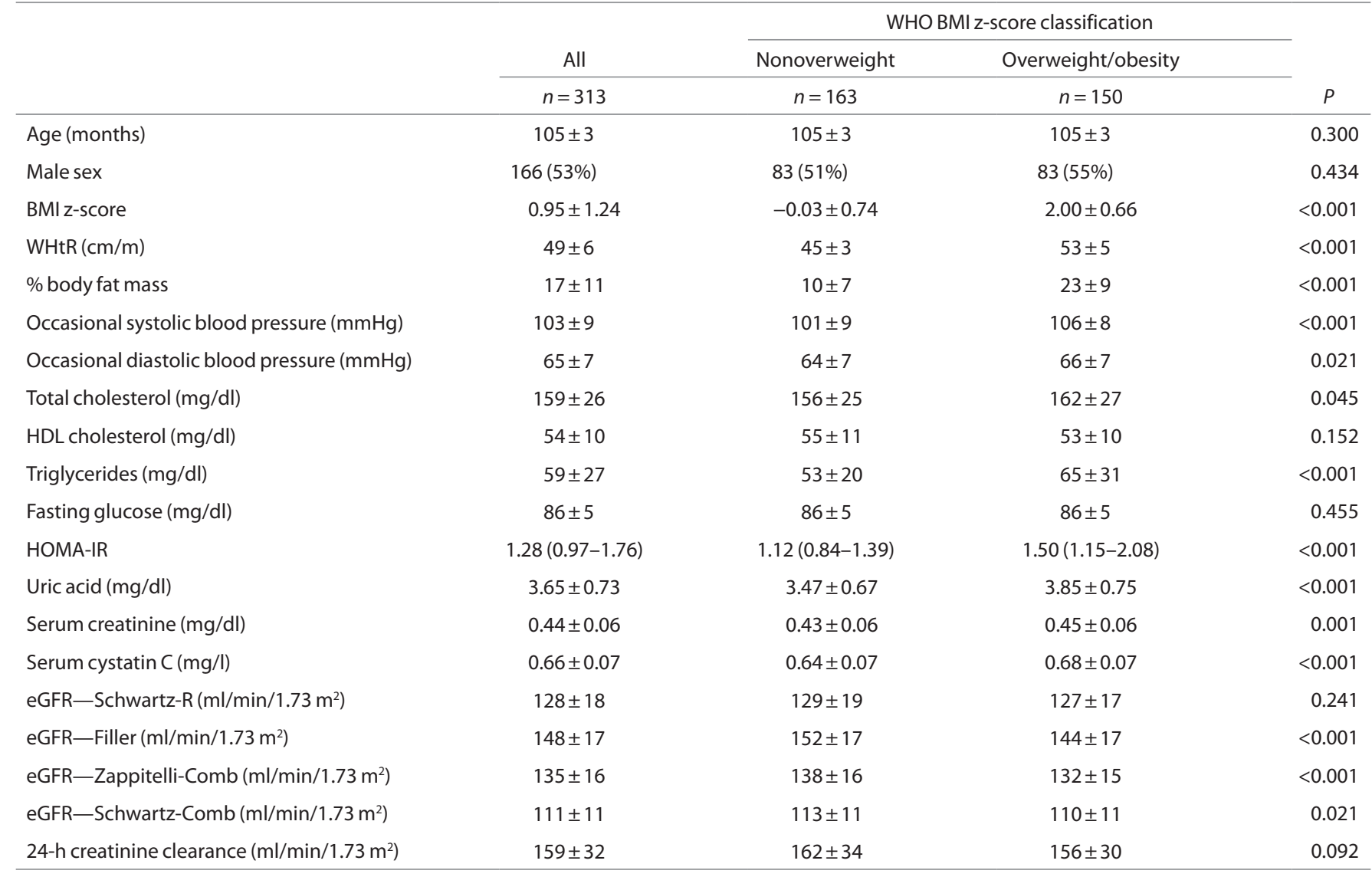

The values presented are mean \pm SD, median (25th-75th percentiles) or $n$ (\%). The nonoverweight group includes children with thinness and normal weight and the overweight/ obesity group includes children with overweight or obesity, according to the WHO classification for BMI z-score (19). Data regarding 24-h creatinine clearance refers only to 298 cases with valid 24-h urine samples.

eGFR, estimated glomerular filtration rate; HDL, high-density lipoprotein; WHO, World Health Organization; WHtR, waist-to-height ratio. 


\section{Articles $\mid$ Correia-Costa et al.}

z-score and eGFR (by Schwartz-R formula or $\mathrm{CrCl}$ ) were independent determinants of CysC (Table 3).

In Table 4, we summarize the agreement between different formulas to estimate eGFR and $\mathrm{CrCl}$. The formula that seemed to have the closest performance to $\mathrm{CrCl}$ (both higher correlation coefficients and higher accuracy values) and to present the most similar results in the nonoverweight and overweight/obesity groups was the ZappitelliComb. The Bland-Altman plots for each formula, using $\mathrm{CrCl}$ as the reference eGFR value, for nonoverweight and overweight/obesity groups, are presented in Figures 2 and 3 , respectively.

\section{DISCUSSION}

In our study of healthy prepubertal children, significant inverse correlations were found between renal function and BMI z-score, with eGFR decreasing significantly with BMI, despite all children having renal function in the normal range. Overweight and obese children had significantly higher levels of both creatinine and CysC and lower eGFR values. a

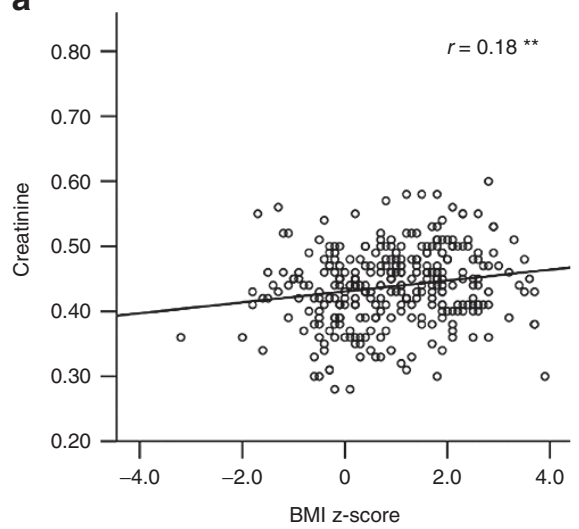

d

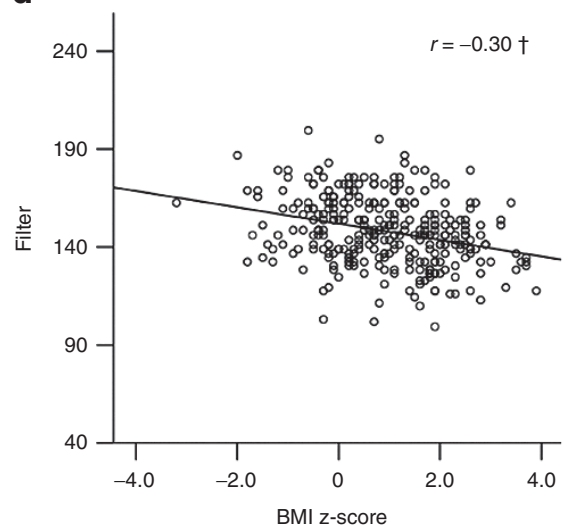

b

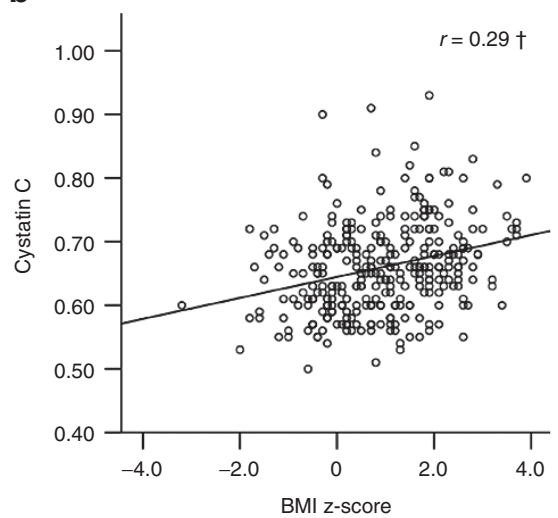

e

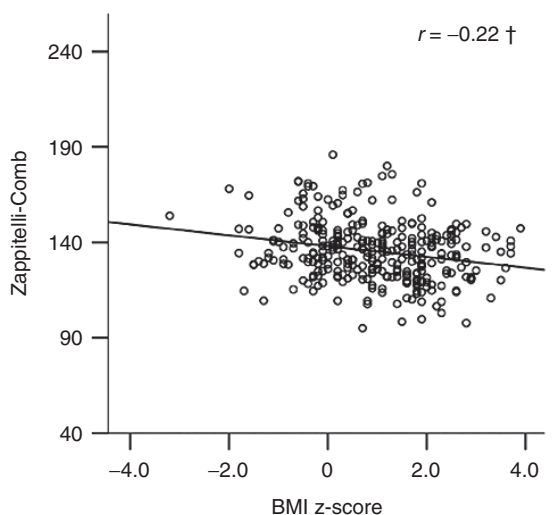

c

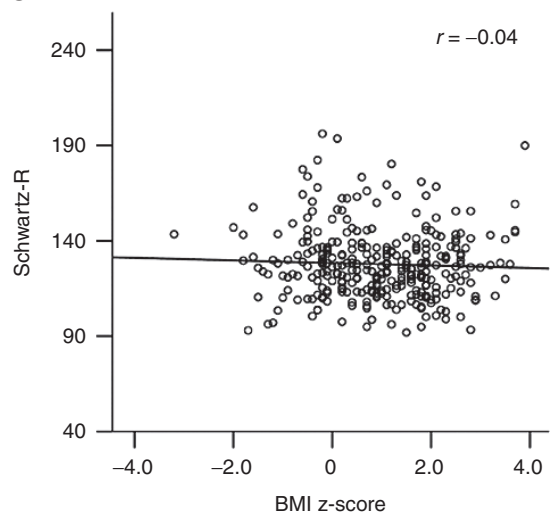

f

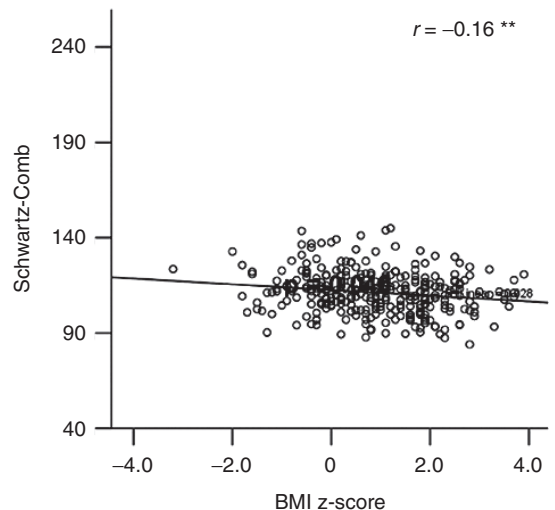

\section{g}

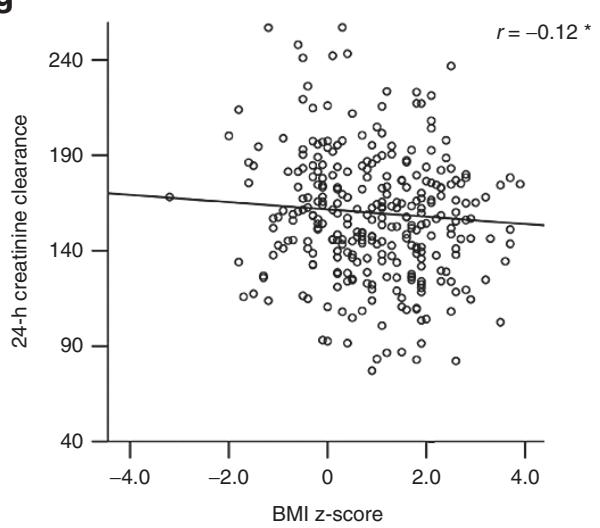

Figure 1. Scatter plots of renal function markers on BMI z-score. Pannels a to $\mathbf{g}$ depict scatter plots of renal function markers on BMI z-score: (a) Creatinine (mg/dl); (b) Cystatin C (mg/l); (c) Revised Schwartz formula (ml/min/1.73 m²); (d) Filler formula (ml/min/1.73 m²); (e) Zappitelli combined formula $\left(\mathrm{ml} / \mathrm{min} / 1.73 \mathrm{~m}^{2}\right) ;(\mathbf{f})$ Schwartz combined formula $\left(\mathrm{ml} / \mathrm{min} / 1.73 \mathrm{~m}^{2}\right) ;(\mathbf{g})$ 24-h creatinine clearance $\left(\mathrm{ml} / \mathrm{min} / 1.73 \mathrm{~m}^{2}\right)$. Data shown are Pearson correlation coefficients $(r)$. Regarding 24 -h creatinine clearance analysis, only 298 cases with valid 24 -h urine samples were included. ${ }^{*} P<0.050$; ${ }^{*} P<0.010 ;{ }^{\dagger} P<0.001$. 


\section{Renal function and obesity in children}

Table 2. Mean changes in renal function markers and estimated glomerular filtration rates per unit of BMI z-score

\begin{tabular}{|c|c|c|c|c|c|c|c|}
\hline & $\begin{array}{l}\text { Creatinine } \\
(\mathrm{mg} / \mathrm{dl})\end{array}$ & $\begin{array}{l}\text { Cystatin C } \\
(\mathrm{mg} / \mathrm{l})\end{array}$ & $\begin{array}{l}\text { eGFR Schwartz-R } \\
\left(\mathrm{ml} / \mathrm{min} / 1.73 \mathrm{~m}^{2}\right)\end{array}$ & $\begin{array}{c}\text { eGFR Filler } \\
\left(\mathrm{ml} / \mathrm{min} / 1.73 \mathrm{~m}^{2}\right)\end{array}$ & $\begin{array}{c}\text { eGFR } \\
\text { Zappitelli-Comb } \\
\left(\mathrm{ml} / \mathrm{min} / 1.73 \mathrm{~m}^{2}\right)\end{array}$ & $\begin{array}{c}\text { eGFR Schwartz- } \\
\text { Comb } \\
\left(\mathrm{ml} / \mathrm{min} / 1.73 \mathrm{~m}^{2}\right)\end{array}$ & $\begin{array}{c}\mathrm{CrCl} \\
\left(\mathrm{ml} / \mathrm{min} / 1.73 \mathrm{~m}^{2}\right)\end{array}$ \\
\hline BMI & 0.007 & 0.017 & -0.51 & -4.26 & -2.77 & -1.10 & -3.54 \\
\hline z-score & $\begin{array}{c}(0.002 \text { to } 0.013) \\
\quad P=0.004\end{array}$ & $\begin{array}{c}(0.011 \text { to } 0.023) \\
P<0.001\end{array}$ & $\begin{array}{c}(-2.17 \text { to } 1.16) \\
P=0.548\end{array}$ & $\begin{array}{c}(-5.77 \text { to }-2.74) \\
P<0.001\end{array}$ & $\begin{array}{c}(-4.16 \text { to }-1.38) \\
P<0.001\end{array}$ & $\begin{array}{c}(-2.01 \text { to }-0.18) \\
P=0.014\end{array}$ & $\begin{array}{c}(-6.52 \text { to }-0.57) \\
P=0.020\end{array}$ \\
\hline
\end{tabular}

The values presented are $\beta$ and 95\% confidence intervals, estimated by multiple linear regression, with renal function markers as dependent variable and BMI z-score (continuous) as independent variable, adjusting for sex and age in months. In the model with 24-h creatinine clearance only 298 cases with valid 24 -h urine samples were included. eGFR, estimated glomerular filtration rate.

Table 3. Mean change in serum cystatin C (mg/l), per unit of BMI z-score, and renal function (assessed either by revised Schwartz formula or 24-h creatinine clearance)

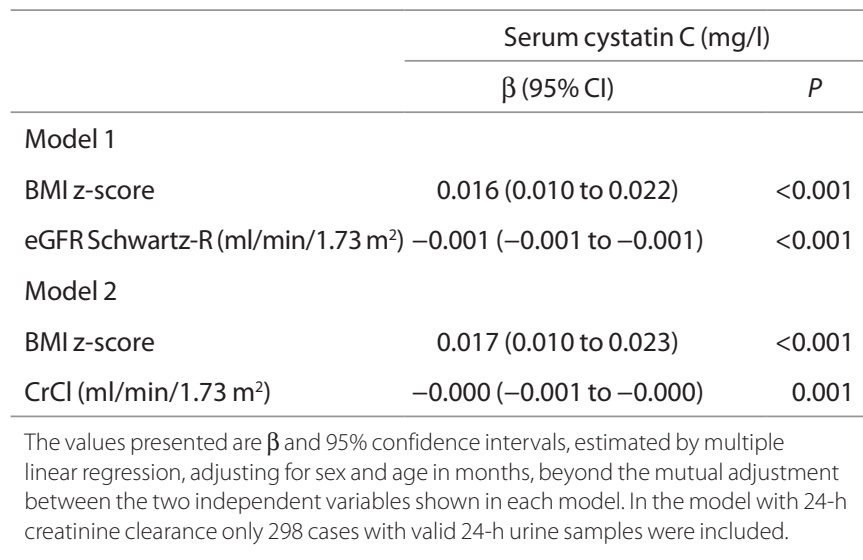

Renal impairment in association with obesity in children is still a debated question and contradictory results continue to emerge. It is well established that in the first stages of renal dysfunction associated with obesity, hyperfiltration occurs as a physiological adaptation of the kidney to the increased body mass $(17,18)$. Concordantly, some authors reported strong positive correlations between measures of obesity and renal eGFR (6), while others did not observe any differences in eGFR levels between overweight/obese and nonoverweight children $(9,10)$. In our sample of young prepubertal children, actually, we found that obesity was associated with lower renal function. Our findings may result from the fact that we had a larger sample of both overweight/obese and nonoverweight children than most of the studies cited. The variation of body composition and eGFR with growth is a major concern in studies that include an age-wide sample; not only did we study a narrow age interval, but also models were adjusted for age (in months). Besides, it is noteworthy that in our sample most of the children in the overweight/obesity group (about $60 \%$ ) already presented overweight or obesity at the age of 4 ; thus longer times of exposure might implicate more advanced stages of obesity-related renal injury, with measurable differences in renal function markers becoming more apparent. At this point we cannot ascertain if a previous phase of hyperfiltration occurred in the initial phases of renal adaptation to higher body fat mass in our sample. Similarly to our results, other authors in large representative population-based cohort studies found lower renal function estimations in obese children $(7,8)$. A large population-based study of adolescents in the United States found significantly lower mean eGFR levels in obese subjects, but the authors considered that obesity did not play a significant role in changing the eGFR distribution in the entire study population (19). The possible mechanisms through which obesity might cause renal impairment in young children are diverse. Obesity-related kidney damage is thought to initiate with a hyperfiltration phase, as previously stated, that later potentiates progressive renal damage, with increased loss of proteins and a last phase of glomerulomegaly, cellular remodeling, and fibrotic scaring (20). More recently, the recognition that adipose tissue is involved in the production of several bioactive molecules, such as adiponectin, leptin, inflammatory, and oxidative stress markers, even in children $(21,22)$, and that many of those substances may affect the cells in the renal glomeruli and cause pathological changes has been an important focus of research (20). It has been speculated that the production of active fibrotic cytokines recognized in several kidney diseases as markers of disease activity and histopathological deterioration, might also be increased in the set of obesity (23). Such substances were shown to be increased in children with congenital single kidney in comparison with controls, even in early phases of hyperfiltration when proteinuria and renal insufficiency are still absent (24). Since kidney diseases with reduced renal mass are believed to mimic what happens in obesity, representing the clinical translation of the experimental hyperfiltration models (25), the increased levels of these substances, promoted in the proinflammatory and pro-oxidant setting of obesity, might partially explain the kidney impairment observed. Moreover, it is important to recognize that some of the conditions associated with obesity might also act to reduce renal function, such as high blood pressure (and glomerular hypertension), hyperlipidemia, insulin resistance, hyperleptinemia and increased sympathetic activity and overactivation of renin-angiotensin system $(2,26)$. Another factor contributing to explain the impact of obesity on the kidney might be the reduced nephron mass associated with conditions like being born small for gestational age or preterm (27). These children are at higher risk of becoming obese, due to prenatal programming, and since the nephron number is fixed at birth, weight gain will increase the metabolic and hemodynamic load on each individual nephron and might contribute to more rapid renal deterioration (25).

We reported that the differences in CysC levels were not exclusively attributable to nonrenal differences, perchance directly related to differences in the body fat mass. Thus, the 


\section{Articles | Correia-Costa et al.}

Table 4. Accuracy and bias of different formulas for glomerular filtration rate estimation compared to $24-\mathrm{h}$ creatinine clearance, in nonoverweight and overweight/obese children (24-h creatinine clearance (mean \pm SD)—nonoverweight: $162 \pm 34 \mathrm{ml} / \mathrm{min} / 1.73 \mathrm{~m}{ }^{2}$; overweight/ obese: $156 \pm 30 \mathrm{ml} / \mathrm{min} / 1.73 \mathrm{~m}^{2}$ )

Equations using either serum creatinine or serum cystatin $C$

\begin{tabular}{|c|c|c|c|c|}
\hline & \multicolumn{2}{|c|}{ Filler } & \multicolumn{2}{|c|}{ Schwartz-R } \\
\hline eGFR $\left(\mathrm{ml} / \mathrm{min} / 1.73 \mathrm{~m}^{2}\right)$, mean $\pm \mathrm{SD}$ & $152 \pm 17$ & $143 \pm 17^{*}$ & $129 \pm 19^{*}$ & $127 \pm 17^{*}$ \\
\hline Accuracy $10 \%(\%)$ & 39.6 & 38.9 & 17.5 & 23.6 \\
\hline $\begin{array}{l}\text { Correlation coefficient between eGFR } \\
\text { and } \mathrm{CrCl}\end{array}$ & 0.148 & $0.288^{* *}$ & $0.622^{* *}$ & $0.469 * *$ \\
\hline
\end{tabular}

Equations using both serum creatinine and CysC

\begin{tabular}{|c|c|c|c|c|}
\hline & \multicolumn{2}{|c|}{ Zappitelli-Comb } & \multicolumn{2}{|c|}{ Schwartz-Comb } \\
\hline & Nonoverweight & Overweight/obesity & Nonoverweight & Overweight/obesity \\
\hline eGFR $\left(\mathrm{ml} / \mathrm{min} / 1.73 \mathrm{~m}^{2}\right)$, mean $\pm \mathrm{SD}$ & $139 \pm 16^{*}$ & $132 \pm 15^{*}$ & $113 \pm 11^{*}$ & $110 \pm 11^{*}$ \\
\hline Accuracy $10 \%(\%)$ & 31.8 & 30.6 & 8.4 & 10.4 \\
\hline $\begin{array}{l}\text { Correlation coefficient between } \\
\text { eGFR and } \mathrm{CrCl}\end{array}$ & $0.509^{* *}$ & $0.478^{* *}$ & $0.417^{* *}$ & $0.345^{* *}$ \\
\hline
\end{tabular}

In the agreement analysis, only 298 cases with valid 24-h urine samples were included. The nonoverweight group includes children with thinness and normal weight and the overweight/obesity group includes children with overweight or obesity, according to the World Health Organization classification for BMI $z$-score (19). ${ }^{*} P<0.001$, Paired $t$-test in comparison with 24 -h creatinine clearance. ${ }^{* *} P<0.001$, Pearson correlation.

eGFR, estimated glomerular filtration rate.

findings of lower renal function estimations in overweight/ obese children likely represent some degree of renal impairment in relation with obesity, already at such a young age. The pertinence of this remark arises from the fact that, although Cys C emerged as an alternative filtration marker less affected by muscle mass and diet and more sensitive in early phases of renal impairment than creatinine, its levels might be affected by nonrenal determinants, such as body fat (13-15). There are a limited number of studies on $\mathrm{CysC}$ levels in overweight and obese children. Our results are in disagreement with the results from the study by Codoñer-Franch et al. (16), which reports that while $\mathrm{CysC}$ associates with several cardiovascular risk factors, the increased $\mathrm{CysC}$ levels could not be attributed to altered renal function, as evaluated by eGFR. In order to clarify the body composition interference in the levels of CysC, the indirect estimations of renal function need to be compared with exact eGFR determinations by exogenous methods and, to our knowledge, such a study does not exist in the general pediatric population.

Although recognizing the lack of a solid reference standard for eGFR in our study, we compared a selection of creatinine- and $\mathrm{Cys} C$-based and combined equations with the $\mathrm{CrCl}$, aiming to better understand their differences in relation with body composition. Several studies in children reported
eGFR formulas to be reliable methods to assess kidney function $(28,29)$ but the accuracy of $\mathrm{CrCl}$ is rarely reported. Since endogenous creatinine clearance has been widely used and is still a frequent proxy used in clinical practice to estimate eGFR, we decided to use it as our reference standard. It has long been recognized that $\mathrm{CrCl}$ can be affected by inaccuracies in the quantity of urine collected and that tubular secretion of creatinine falsely elevates the eGFR estimation (30). It is important to emphasize that on top of a strict protocol for field work, we carefully assessed the quality of 24-h urine samples, excluding $5 \%$ of our samples. We found that, both in nonoverweight and overweight/obese children, eGFR estimation using Zappitelli-Comb formula revealed to be the closest to the $\mathrm{CrCl}$. A recent study in adults addressed the issue of possible differential performances of eGFR equations, either including creatinine, $\mathrm{Cys} C$ or both, in subgroups defined by body composition (31). The authors reported that, in comparison with exogenous methods for eGFR determination, both creatinine- and CysC-based equations underestimated eGFR in people with higher BMI and that the higher absolute differences were found in people with normal renal function (eGFR $\left.>90 \mathrm{ml} / \mathrm{min} / 1.73 \mathrm{~m}^{2}\right)$. The absolute differences and bias among subgroups were smallest for combined equations and the authors advocated towards the preferential use of combined 
a

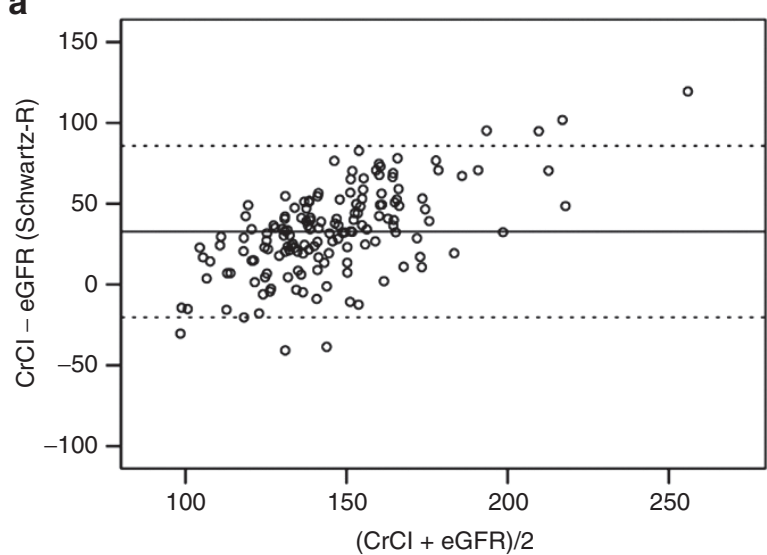

C

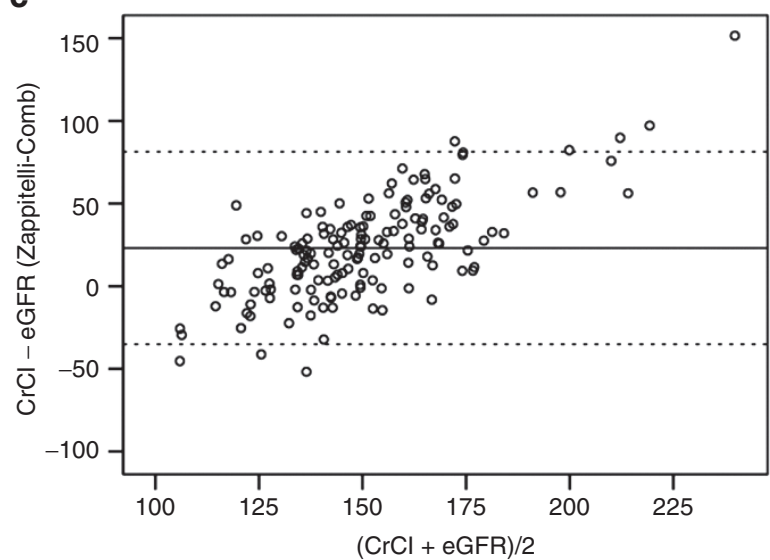

b

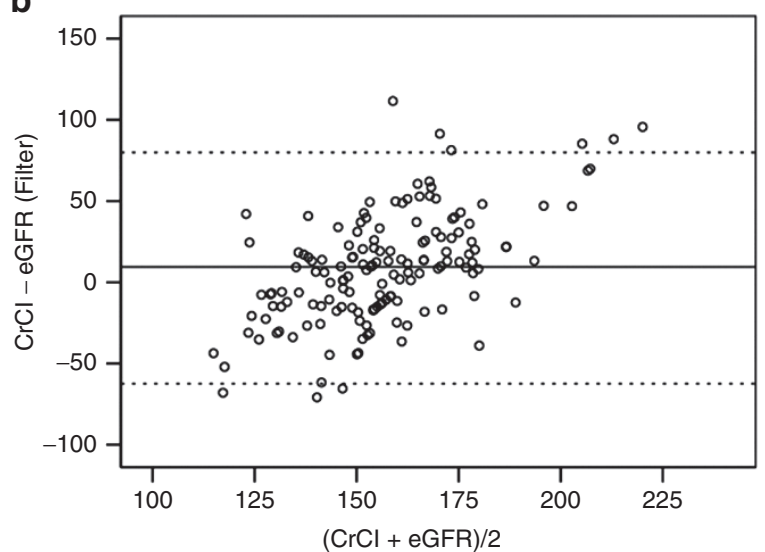

d

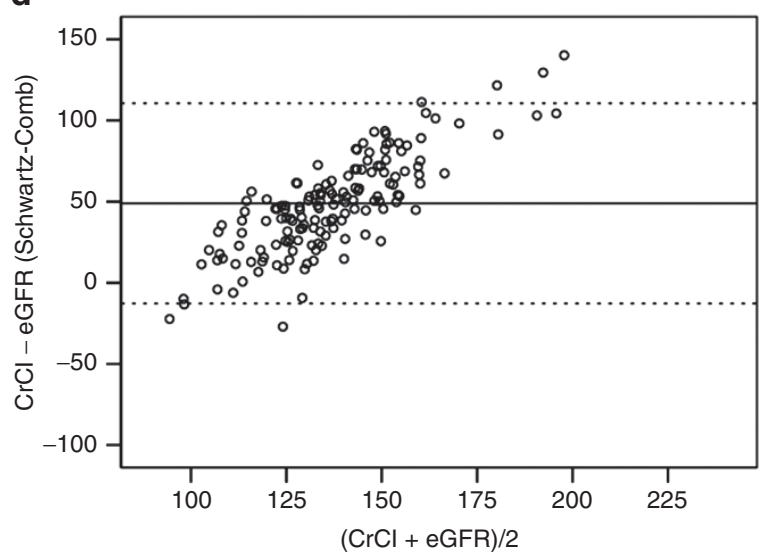

Figure 2. Bland-Altman plots of the different formulas for glomerular filtration rate estimation in comparison with 24-h urine creatinine clearance, for nonoverweight subjects. Pannels a to $\mathbf{d}$ depict Bland-Altman plots of the different formulas for glomerular filtration rate estimation in comparison with 24-h urine creatinine clearance, for nonoverweight subjects: (a) Revised Schwartz formula; (b) Filler formula; (c) Zappitelli combined formula; (d) Schwartz combined formula. In the agreement analysis, only 298 cases with valid 24-h urine samples were included. The horizontal full line represents the mean difference between 24-h creatinine clearance and eGFR, and the horizontal dashed lines the limits of agreement. The nonoverweight group includes children with normal weight, according to the World Health Organization classification for BMI z-score (35).

formulas. These findings are in line with studies that found a good agreement of Zappitelli-Comb formula and exogenous eGFR determinations $(28,29)$ and are concordant with the recommendation to prefer combined formulas, especially in subgroups in which body composition might interfere (31). The use of creatinine and CysC in combination seems to result in improved accuracy, with the effects of the non-GFR determinants of each marker being more attenuated than when using each one alone. In conclusion, the use of combined formulas to estimate eGFR might represent a valid alternative to the lengthy and difficult collection of 24-h urine in children, both in nonoverweight and overweight/obese children.

The major strength of our study is the large sample of overweight and obese children, otherwise healthy, with very complete data on cardiovascular and renal markers, allowing an extensive and thorough analysis. The lack of repeated measurements of kidney function might be a limitation to putatively reduce short-term fluctuations due to external factors, but we believe that this was, at least, partly overcome by the use of multiple methods for renal function assessment. Besides, we selected eGFR formulas taking into account the laboratory methods for renal markers determination used in our center and used in the validation of each formula, thus reducing the systematic bias between the methods and allowing comparisons. Serum creatinine was determined by the Jaffé compensated technique but calibrated according to international parameters (32), thus allowing comparison with formulas that used creatinine measured by an enzymatic assay, such as Schwartz-R, Zappitelli-Comb, and Schwartz-Comb. The comparison of several methods in overweight/obese and nonoverweight children is of major interest, since in clinical practice, when observing healthy children, we need to be able to decide which method might be reliably used across all groups of body composition. Apparently the 24-h urine collection might be dispensable if both creatinine and CysC levels are available.

We acknowledge some limitations in our study. First, an exogenous method would be important when aiming to determine the accuracies of indirect measurements of renal function and to definitely characterize the degree of actual renal impairment in overweight/obese subjects with higher CysC levels. Second, the cross-sectional design of the present analysis, along with the fact that renal injury markers should be 
a

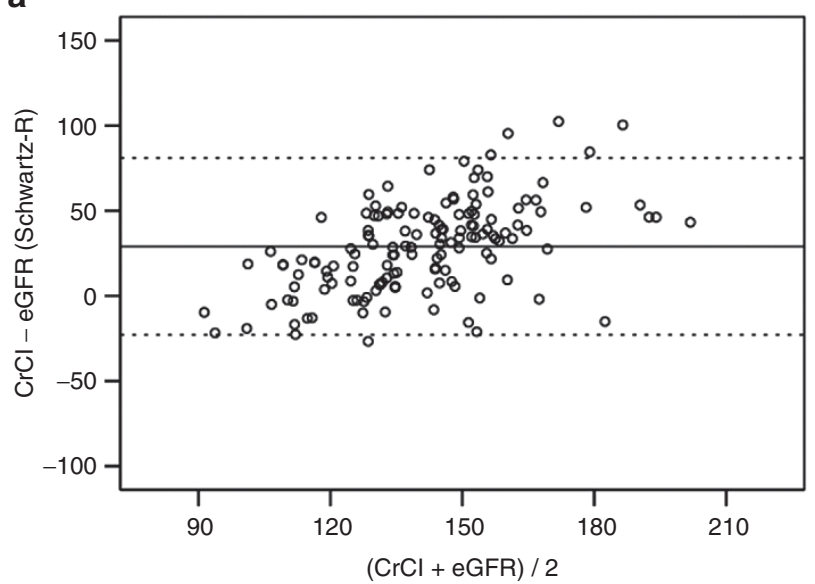

c

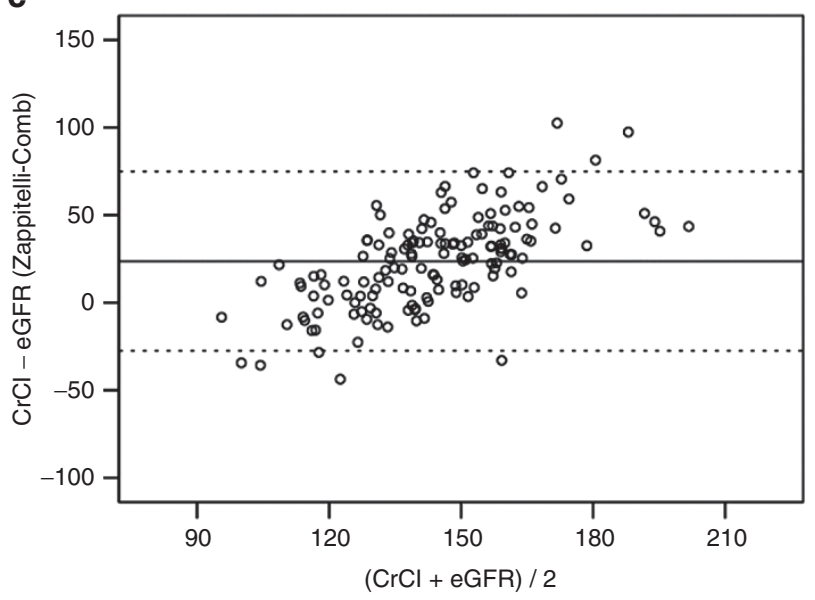

b

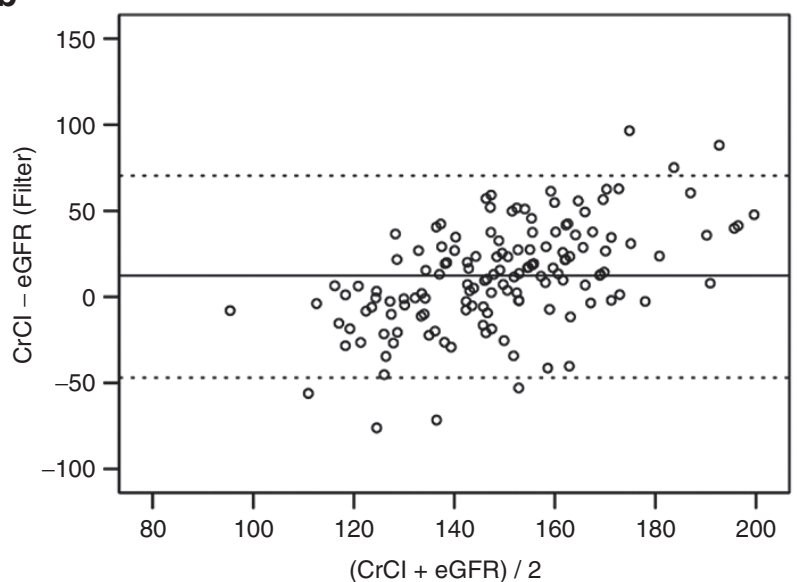

d

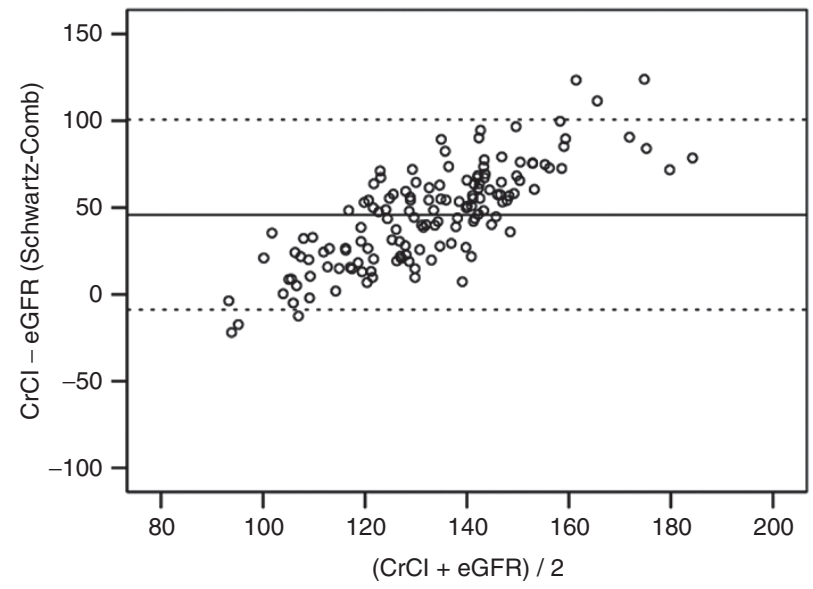

Figure 3. Bland-Altman plots of the different formulas for glomerular filtration rate estimation in comparison with 24-h urine creatinine clearance, for overweight/obese subjects. Pannels a to $\mathbf{d}$ depict Bland-Altman plots of the different formulas for glomerular filtration rate estimation in comparison with 24-h urine creatinine clearance, for overweight/obese subjects: (a) Revised Schwartz formula; (b) Filler formula; (c) Zappitelli combined formula; (d) Schwartz combined formula. In the agreement analysis, only 298 cases with valid 24-h urine samples were included. The horizontal full line represents the mean difference between 24-h creatinine clearance and eGFR, and the horizontal dashed lines the limits of agreement. The overweight/obese group includes children with overweight and obesity, according to the World Health Organization classification for BMI z-score (35).

investigated in the long-term, is also a limitation. Nevertheless, this limitation can be overcome in the future if, as intended, we follow these children through adulthood in order to better understand the real impact of obesity on their kidney function.

\section{Conclusion}

In conclusion, we report that young prepubertal children with overweight or obesity at the age of $8-9$ y already present significantly lower eGFR. We showed that CysC levels are increased in overweight/obese children and that this elevation most probably represents some degree of renal involvement in the complex net of deleterious effects associated with obesity. The association of obesity with cardiovascular risk factors and lower eGFR levels alerts us to the fact that overweight and obese children are at risk for cardiovascular events and chronic kidney disease. Nevertheless, since our results focus on a very young age when all children still have normal renal function, one can expect to be able to reduce the burden of chronic kidney disease in the adult population if measures against obesity are implemented early in life.

\section{METHODS}

\section{Study Design and Sample}

We conducted a cross-sectional study of children aged 8-9 y that have been followed since birth in a previously established cohort study (Generation XXI) (33). Subjects from the original cohort were eligible for the present study protocol (ObiKid project) if they had anthropometric data and a blood sample withdrawn at the 7-y-old evaluation $(n=4,590)$. We aimed to include a minimum sample of 300 children for the Obikid project's main objective; assuming that about $35 \%$ would be excluded due to refusal to participate, exclusion criteria or incomplete information, 463 children were preselected to be consecutively screened according to the date of their 7-y-old evaluation: 16 could not be contacted, 32 refused to participate, 23 were unable to schedule the study visits during the recruitment period, and 68 met exclusion criteria (4 chronic diseases, 1 chronic usage of medication, 51 living far from the study site, and 12 twins). We finally enrolled 324 participants, between August 2013 and August 2014. For the present analysis, 11 children were additionally excluded due to absence of blood or 24-h urine samples and 313 children were finally included. This sample size would provide a statistical power of $86 \%$ to detect a difference in eGFR, between nonoverweight and overweight/obese children, of at least $8 \mathrm{ml} / \mathrm{min} / 1.73 \mathrm{~m}^{2}$, assuming SD of 24 and $22 \mathrm{ml} / \mathrm{min} / 1.73 \mathrm{~m}^{2}$ in each group, respectively (7). 


\section{Data Collection and Variable Definition}

The study visits took place at the Department of Clinical Epidemiology, Predictive Medicine and Public Health, Faculty of Medicine of the University of Porto. Anthropometric and general physical examinations were performed, according to standard procedures and as previously reported (34). Waist circumference (measured at the umbilicus' line) was indexed to height (WHtR in $\mathrm{cm} / \mathrm{m}$ ) for analysis. Body fat percentage was assessed by foot-to-foot bioelectrical impedance analysis (TBF-300 (Tanita, Arlington Heights, Illinois)). BMI was calculated and BMI-for-age values were classified according to the World Health Organization reference data for BMI z-score into the following categories: nonoverweight $(\leq+1 \mathrm{SD}$, including children with thinness $(n=1)$ and normal weight) and overweight/obesity ( $>1$ SD, including children with overweight and obesity) (35).

Office blood pressure was evaluated with oscillometric validated sphygmomanometers (Elite 92125 (Medel, Parma, Italy)) with an adequately sized cuff in the right arm, by a nonphysician trained interviewer, three times with a 5-min interval, and the second and third measurements were averaged for analysis.

\section{Laboratory Procedures}

Venous blood samples were collected after an overnight fast of at least $8 \mathrm{~h}$ and analyzed for creatinine, CysC, urea, glucose, insulin, and lipids. Insulin resistance was determined using the homeostasis model assessment index (36). All participants collected a 24-h urine sample, which was analyzed for creatinine. All the standard laboratory analyzes were performed in the Clinical Pathology Department of Centro Hospitalar São João, Porto - Portugal.

\section{Renal Function Parameters}

Serum creatinine assay was based on the Jaffé compensated traceable to an isotope dilution mass spectrometry method (Olympus AU 5400 analyzer (Beckman-Coulter, Brea, CA) (32). Urinary creatinine was determined with the same clinical chemistry analyzer. Serum CysC was assayed using a particle-enhanced immunonephelometric assay (N latex CysC (Siemens, Erlangen, Germany).

To estimate eGFR, in $\mathrm{ml} / \mathrm{min} / 1.73 \mathrm{~m}^{2}$, the following formulas were used: revised Schwartz formula (Schwartz-R), $k \times$ (height $(\mathrm{cm}) /$ serum creatinine (mg/dl)); using a $k$ constant of 0.413) (30); Filler formula, $\log ($ GFR $)=1.962+(1.123 \times \log (1 /$ cystatin C $(\mathrm{mg} / \mathrm{l}))(37)$; combined Zappitelli formula (Zappitelli-Comb), $\left(507.76 \times \mathrm{e}^{0.003 \times \text { height }(\mathrm{cm})}\right) /($ cystatin $\mathrm{C}(\mathrm{mg} / \mathrm{l})^{0.635} \times$ serum creatinine $\left.\left.(\mathrm{mg} / \mathrm{dl})^{0.547}\right)\right)(38)$ and combined Schwartz formula (Schwartz-Comb), $39.8 \times$ (height $(\mathrm{cm}) /$ serum creatinine $(\mathrm{mg} / \mathrm{dl}))^{0.456} \times\left(1.8 /\right.$ cystatin C $(\mathrm{mg} / \mathrm{l})^{0.418} \times(30 /$ blood urea nitrogen $)^{0.079} \times(\text { height }(\mathrm{cm}) / 1.4)^{0.179}(\times 1.076$ if females $\left.)\right)(39)$.

All children's parents received information on the correct methods of 24-h urine collection and, upon sample delivery, compliance was rechecked by a brief questionnaire. The samples were considered valid if urinary creatinine was within the range of $11.3-28.0 \mathrm{mg} / \mathrm{kg} / \mathrm{d}$ (according to age- and sex-specific reference values (40)) and if urinary volume was over $300 \mathrm{ml}$; based on these criteria, 15 urine samples were excluded from the analysis. The $\mathrm{CrCl}$ was calculated according to the standard formula and normalized to $1.73 \mathrm{~m}^{2}$ of body surface; body surface area was determined by the Haycock formula (41).

\section{Ethics}

The ObiKid project was approved by the Ethics Committee of Centro Hospitalar São João, Porto-Portugal and Faculty of Medicine of the University of Porto and complies with the Helsinki Declaration. Written informed consent from parents and verbal assent from children was obtained, concerning information, and biological samples gathering.

\section{Statistical Analysis}

Standard statistical analysis was performed using IBM SPSS Statistics for Windows, Version 20.0 (Chicago, IL). Differences between groups in continuous variables were evaluated with Student's $T$-tests or Mann-Whitney tests. Bivariate associations were assessed by Pearson correlation tests. Linear regression models were used to estimate mean adjusted changes in renal function per unit of BMI z-score. Two-sided $P<0.05$ was considered significant. To evaluate the agreement between methods of eGFR estimation, calculation of differences (means $\pm \mathrm{SD}$ ), accuracies (within 10,30, and 50\%), and absolute bias (i.e., average difference between eGFR and $\mathrm{CrCl}$ ) were performed and Bland Altman charts were plotted.

\section{ACKNOWLEDGMENTS}

The authors gratefully acknowledge the contribution of all members of the research team and staff of Generation XXI.

\section{STATEMENT OF FINANCIAL SUPPORT:}

This project was supported by FEDER funds from Programa Operacional Factores de Competitividade - COMPETE (FCOMP-01-0124-FEDER-028751) and by the Portuguese Foundation for Science and Technology, Lisbon, Portugal (PTDC/DTP-PIC/0239/2012), that granted the funds for study design and data collection and analysis. Franz Schaefer was supported by the European Renal Association - European Dialysis and Transplant Association Research Programme, Parma, Italy and the KfH Foundation for Preventive Medicine, Neu-Isenburg, Germany. L.C.-C. was supported by the Portuguese Foundation for Science and Technology (grant SFRH/SINTD/95898/2013).

Disclosure: The authors have nothing to disclose.

\section{REFERENCES}

1. Hall JE, Crook ED, Jones DW, Wofford MR, Dubbert PM. Mechanisms of obesity-associated cardiovascular and renal disease. Am J Med Sci 2002;324:127-37.

2. Kalaitzidis RG, Siamopoulos KC. The role of obesity in kidney disease: recent findings and potential mechanisms. Int Urol Nephrol 2011;43: 771-84.

3. Hsu CY, McCulloch CE, Iribarren C, Darbinian J, Go AS. Body mass index and risk for end-stage renal disease. Ann Intern Med 2006;144:21-8.

4. Wang Y, Chen X, Song Y, Caballero B, Cheskin LJ. Association between obesity and kidney disease: a systematic review and meta-analysis. Kidney Int 2008;73:19-33.

5. Savino A, Pelliccia P, Chiarelli F, Mohn A. Obesity-related renal injury in childhood. Horm Res Paediatr 2010;73:303-11.

6. Koulouridis E, Georgalidis K, Kostimpa I, Koulouridis I, Krokida A, Houliara D. Metabolic syndrome risk factors and estimated glomerular filtration rate among children and adolescents. Pediatr Nephrol 2010;25:491-8.

7. Duzova A, Yalçinkaya F, Baskin E, Bakkaloglu A, Soylemezoglu O. Prevalence of hypertension and decreased glomerular filtration rate in obese children: results of a population-based field study. Nephrol Dial Transplant 2013;28 Suppl 4:iv166-71

8. Soylemezoglu O, Duzova A, Yalçinkaya F, Arinsoy T, Süleymanlar G. Chronic renal disease in children aged $5-18$ years: a population-based survey in Turkey, the CREDIT-C study. Nephrol Dial Transplant 2012;27 Suppl 3:iii146-51.

9. Goknar N, Oktem F, Ozgen IT, et al. Determination of early urinary renal injury markers in obese children. Pediatr Nephrol 2015;30:139-44.

10. Cindik N, Baskin E, Agras PI, Kinik ST, Turan M, Saatci U. Effect of obesity on inflammatory markers and renal functions. Acta Paediatr 2005;94:1732-7.

11. Schwartz GJ, Work DF. Measurement and estimation of GFR in children and adolescents. Clin J Am Soc Nephrol 2009;4:1832-43.

12. Roos JF, Doust J, Tett SE, Kirkpatrick CM. Diagnostic accuracy of cystatin $\mathrm{C}$ compared to serum creatinine for the estimation of renal dysfunction in adults and children-a meta-analysis. Clin Biochem 2007;40:383-91.

13. Naour N, Fellahi S, Renucci J-F, et al. Potential contribution of adipose tissue to elevated serum cystatin C in human obesity. Obesity 2009;17: 2121-6.

14. Muntner P, Winston J, Uribarri J, Mann D, Fox CS. Overweight, obesity, and elevated serum cystatin C levels in adults in the United States. Am J Med 2008;121:341-8.

15. Vupputuri S, Fox CS, Coresh J, Woodward M, Muntner P. Differential estimation of CKD using creatinine- versus cystatin C-based estimating equations by category of body mass index. Am J Kidney Dis 2009;53: 993-1001.

16. Codoñer-Franch P, Ballester-Asensio E, Martínez-Pons L, VallecilloHernández J, Navarro-Ruíz A, del Valle-Pérez R. Cystatin C, cardio- 


\section{Articles $\mid$ Correia-Costa et al.}

metabolic risk, and body composition in severely obese children. Pediatr Nephrol 2011;26:301-7.

17. Tomaszewski M, Charchar FJ, Maric C, et al. Glomerular hyperfiltration: a new marker of metabolic risk. Kidney Int 2007;71:816-21.

18. Chagnac A, Weinstein T, Korzets A, Ramadan E, Hirsch J, Gafter U. Glomerular hemodynamics in severe obesity. Am J Physiol Renal Physiol 2000;278:F817-22.

19. Fadrowski JJ, Neu AM, Schwartz GJ, Furth SL. Pediatric GFR estimating equations applied to adolescents in the general population. Clin J Am Soc Nephrol 2011;6:1427-35.

20. Mathew AV, Okada S, Sharma K. Obesity related kidney disease. Curr Diabetes Rev 2011;7:41-9.

21. Mauras N, Delgiorno C, Kollman C, et al. Obesity without established comorbidities of the metabolic syndrome is associated with a proinflammatory and prothrombotic state, even before the onset of puberty in children. J Clin Endocrinol Metab 2010;95:1060-8.

22. Codoner-Franch P, Boix-Garcia L, Simo-Jorda R, et al. Is obesity associated with oxidative stress in children? Int J Pediatr Obes 2010;5:56-63.

23. Prunotto M, Ghiggeri G, Bruschi M, et al. Renal fibrosis and proteomics: current knowledge and still key open questions for proteomic investigation. J Proteomics 2011;74:1855-70.

24. Wasilewska A, Zoch-Zwierz W, Taranta-Janusz K. Urinary transforming growth factor betal in children and adolescents with congenital solitary kidney. Pediatr Nephrol 2009;24:753-9.

25. Cignarelli M, Lamacchia O. Obesity and kidney disease. Nutr Metab Cardiovasc Dis 2007;17:757-62.

26. Yoshida T, Takei T, Shirota S, et al. Risk factors for progression in patients with early-stage chronic kidney disease in the Japanese population. Intern Med 2008;47:1859-64.

27. Abitbol CL, Chandar J, Rodríguez MM, et al. Obesity and preterm birth: additive risks in the progression of kidney disease in children. Pediatr Nephrol 2009;24:1363-70.

28. Bacchetta J, Cochat P, Rognant N, Ranchin B, Hadj-Aissa A, Dubourg L. Which creatinine and cystatin $C$ equations can be reliably used in children? Clin J Am Soc Nephrol 2011;6:552-60.

29. Andersen TB, Eskild-Jensen A, Frøkiaer J, Brøchner-Mortensen J. Measuring glomerular filtration rate in children; can cystatin $\mathrm{C}$ replace established methods? A review. Pediatr Nephrol 2009;24:929-41.
30. Schwartz GJ, Muñoz A, Schneider MF, et al. New equations to estimate GFR in children with CKD. J Am Soc Nephrol 2009;20:629-37.

31. Fan L, Inker LA, Rossert J, et al. Glomerular filtration rate estimation using cystatin $\mathrm{C}$ alone or combined with creatinine as a confirmatory test. Nephrol Dial Transplant 2014;29:1195-203.

32. Myers GL, Miller WG, Coresh J, et al.; National Kidney Disease Education Program Laboratory Working Group. Recommendations for improving serum creatinine measurement: a report from the Laboratory Working Group of the National Kidney Disease Education Program. Clin Chem 2006;52:5-18.

33. Larsen PS, Kamper-Jørgensen M, Adamson A, et al. Pregnancy and birth cohort resources in europe: a large opportunity for aetiological child health research. Paediatr Perinat Epidemiol 2013;27:393-414.

34. Durão C, Severo M, Oliveira A, et al. Evaluating the effect of energy-dense foods consumption on preschool children's body mass index: a prospective analysis from 2 to 4 years of age. Eur J Nutr 2014;54:835-43.

35. de Onis M, Onyango AW, Borghi E, Siyam A, Nishida C, Siekmann J. Development of a WHO growth reference for school-aged children and adolescents. Bull World Health Organ 2007;85:660-7.

36. Matthews DR, Hosker JP, Rudenski AS, Naylor BA, Treacher DF, Turner RC. Homeostasis model assessment: insulin resistance and betacell function from fasting plasma glucose and insulin concentrations in man. Diabetologia 1985;28:412-9.

37. Filler G, Lepage N. Should the Schwartz formula for estimation of GFR be replaced by cystatin C formula? Pediatr Nephrol 2003;18:981-5.

38. Zappitelli M, Parvex P, Joseph L, et al. Derivation and validation of cystatin C-based prediction equations for GFR in children. Am J Kidney Dis 2006;48:221-30.

39. Schwartz GJ, Schneider MF, Maier PS, et al. Improved equations estimating GFR in children with chronic kidney disease using an immunonephelometric determination of cystatin C. Kidney Int 2012;82: 445-53.

40. Remer T, Neubert A, Maser-Gluth C. Anthropometry-based reference values for 24-h urinary creatinine excretion during growth and their use in endocrine and nutritional research. Am J Clin Nutr 2002;75:561-9.

41. Haycock GB, Schwartz GJ, Wisotsky DH. Geometric method for measuring body surface area: a height-weight formula validated in infants, children, and adults. J Pediatr 1978;93:62-6. 